\title{
The Role of Individual Difference Factors among Early, Middle and Late Immersion Undergraduate ESL Learners
}

\author{
Olusiji Lasekan \\ Universidad Católica de Temuco \\ Temuco, Chile
}

\begin{abstract}
Numerous investigations on the impact of individual difference factors on second language learning are being carried out to improve the effectiveness and productivity of the language learningteaching process. Therefore, with the recent categorization of ESL learners at Indian colleges and universities into early, middle and late immersion students, this study examines the effect of individual difference factors (self-efficacy, anxiety, willingness to communicate, culture, human (peers', teachers' and parents' motivational influence) and their interrelatedness with the learners' motivational intensity as well as English proficiency on each group of immersion learners. A structured, self-report questionnaire was administered to over three hundred and fifty-eight students in different colleges. Participants were classified into different immersion groups based on the number of years they had studied either in English or in Vernacular medium high school. Apart from low motivational intensity recorded among early immersion students, the inferential statistics show a moderate positive correlation between motivational intensity and other motivational variable factors such as self-confidence and human (teachers', parents' and peers' motivational influence). The correlational analysis also suggests the positive impact of self-confidence and intrinsic motivation on learners' English proficiency enhancement. I discuss several potential interpretations for findings and implications for L2 language pedagogy and research.
\end{abstract}

Keywords: India; Immersion; Individual difference factors; Motivational intensity; English proficiency.

\section{Introduction}

As far as second language (L2) education is concerned, a large volume of published studies has established the significant role of Individual Difference (ID) factors in the facilitation of the L2 learning process. Over the years, scholars are adding to the growing list of ID factors. As of 1993, anxiety, motivation, self- 
esteem, risk-taking, language learning styles, tolerance of ambiguity, and gender had been identified as critical variables (Oxford \& Ehrman, 1993). By 2002, intelligence, language aptitude, and age were added to the list (Robinson, 2002). A recent study sheds light on the impact of socioeconomic status (Gayton, 2010) and cognitive variables such as memory (Kidd, Donnelly, \& Christiansen, 2018) on L2 learning. Among all these ID factors, learners' motivation has been the focus of many researchers and practitioners. The importance of motivation can be accrued to its significant effect on the success of learning not only in L2 instruction but in every field of education (Hiromori, Matsumoto \& Nakayama, 2012).

Regarding the role of ID among undergraduates who are learning English as a foreign language (EFL), extensive studies have been conducted in different contexts. In the Chilean context, Gregersen (2000) research shows how ID factors influence the level of English proficiency among pre-service English teachers. In Saudi Arabia, anxiety, motivation, and self-efficacy were found to have a positive impact on learners' English proficiency (Aljasir, N, 2016). Despite this interest, no previous research has investigated a longitudinal study in this area in India. This dimension of ID factors research is needed to solve the disparity problem in English proficiency at the undergraduate level between students who had studied in Regional and English Medium of High School (EMHS). While the latter is a High school where English is taught both as a language and across all curriculums, the former teaches English as a second language and other degree courses in their respective mother tongues (Anand, 1971). As English is the medium of instruction across the curriculum at higher institutions, students from Regional Medium of High School (RMHS) have been found to perform poorly both in English and other academic subjects at this level. This low performance causes many of them to suffer psychological trauma. Cases of taunting, teasing, and segregation by their counterparts from EMS for their low performance in a combined English undergraduate class have been widely reported (Johari, 2015). To identify different groups of English learners in Indian universities, a recent study from the country has identified three different groups of English learners (Lasekan, 2019). These groups are early, middle, and late immersion undergraduate ESL learners. The author's categorization is based on the number of years every student had studied prior to and after admission into the university. Early immersion students are students that start learning English across the curriculum between class 1 and class 10, which comprises both primary and high school educations. This group of learners would have learned English across the curriculum for at least five years by the time they complete the second year of their university education. Middle immersion students are a group of students that begin English immersion from class 11, which is the beginning of Pre-university school. Such students would have learned English across the curriculum for four years by the time they complete the second year of their university education. Late immersion students are the category of students that starts learning English from the first year of their university education. They would have learned English across the curriculum for two years by the time they finish the second year of their university education. 
Having established the existence of different groups of English learners, it is imperative to question the roles of ID factors in L2 learning among them. Therefore, the purpose of this study is to identify individual difference factors that have a strong influence on early, middle, and late immersion undergraduate students. Also, determine the degree of relationships between ID factors (anxiety, self-efficacy, Willingness to Communicate, parents, teachers, peers, motivational intensity, and cultural interest) and English proficiency. This study is expected to help education policymakers, curricula designers, teachers, and researchers to understand factors that affect students' motivation and attitude towards learning English. This research will also aid them as practitioners to make an informing decision on how to improve the quality of English language teaching in classrooms.

\section{Literature Review}

According to Gardner, one of the pioneers of research in L2 learning motivation described motivation as a combination of three components, which includes effort, desire to achieve the goal of learning the language, and a favorable attitude towards learning the language (Gardner, 1985). Out of these three components, motivational intensity as a component has been stressed extensively in the body of literature because effort as a standard measure of motivation has been identified as the pivotal needed by any L2 learner to succeed in learning the target language successfully (Pintrich \& Schunk, 2002).

Gardner et al. (1999) posited that the notion of motivational intensity is defined as the goal-directed level of effort and persistence that learners expend towards learning a foreign language. In support of the significance of motivational intensity, Ely (1986) noted that before researchers attempt to understand learner's motives and motivation, it is crucial to determine and understand the degree of effort that learners intend to expend in learning the foreign language.

Copious of studies on the level or pattern of motivational intensity change between or among different groups of learners have been investigated. For instance, in an attempt to understand the level of motivational intensity between Japanese high school students and undergraduate students, Shibuya (2008) work indicates that the motivational intensity at which undergraduate students learn and study English dropped drastically in the first year they got admitted into the university. The sharp decline is attributed to the change of priority of what to learn at the undergraduate level. They are of the view that since the passing of an English exam is only needed to gain admission into the university, then it is not essential to spend so much time learning it once the goal is achieved. In a major study to establish differences between motivation and motivational intensity, the work of Firdosfida \& Ravindra (2016) revealed that Omani English learners have high motivation. Still, they have a lower motivational intensity to learn the language. In other words, a learner can be highly motivated to learn English, but they are not ready to expend effort and time to learn the language. Having established the significance of motivational intensity in second language learning, a more experimental study is required at the different entry-level of immersions in India. Therefore, empirical research is needed to understand the 
level of motivational intensity among early, middle, and late immersion students.

A considerable amount of literature has been published on the relationship between motivational intensity and other ID factors (Guilloteaux \& Dornyei, 2008; Brown, Robson \& Rosenkjar, 2001). Regarding the role of human factors on learners' motivational intensity, the impact of teachers' motivational strategies on learners' motivation was analyzed (Guilloteaux \& Dornyei, 2008). Peer tutoring was found to have a positive influence on students' motivation (Alrajhi, \& Aldhafri, 2015) and positive correlation between parental encouragement and motivation was reported (Henkel, 2009). Concerning the impact of anxiety on motivation, a negative correlation is widely reported among L2 learners (Brown et al., 2001: Khodadady \& Khajavy, 2013). Considering the importance of communicative competence in L2 learning, previous studies have succeeded in investigating learners' WTC in the target language, especially as it relates to motivation. Based on past works in this area, the degree of correlation between the two variables is a function of the level of motivation itself. For instance, a study conducted among Spanish undergraduate EFL learners showed a positive correlation between their motivation to learn English and their WTC in the language (Lahuerta, 2014). Variables that are positively correlated with motivational intensity are instrumental motivation (Firdosfida \& Ravindra, 2016) and positive attitude towards foreign language (Oroujlou \& Vahedi, 2011).

According to Basta (2011), it is the ability level of a person to use a language both for basic communication with others and academic purposes. In other words, it is the application of these four skills in both socio-cultural and educational settings. Numerous studies conducted in different contexts have found a strong link between English proficiency and different individual difference factors. Studies have identified teachers' motivational strategies in classroom (Dornyei, 2001), peer-assisted learning (Fuchs et al., 1997) and parental level of education (Entwisle, \& Astone, 1994), as key human factors that influence learners' English proficiency. Whereas, other than anxiety (Andrade \& Williams, 2009), a high level of self-confidence (Satriani, 2014), WTC (McCroskey \& Richmond, 1990) and intrinsic motivation (Ababneh, 2013) are linked to the fostering of learners' English proficiency.

Having review few literatures, an attempt has been made to articulate how this study is situated at the crossroads of three frameworks, one ID factors, English proficiency, and one contextual (immersion education). The overlap between these three frameworks has shaped the conceptualization of this study and it shapes how to proceed with the analysis and the presentation of the findings.

\section{Methods}

This study adopted a correlational research design, which measures the level of association between two or more scores or between two or more variables that have been obtained from the same group of subjects. Correlation research was used to predict and describe the association between English proficiency and motivational intensity, as well as other ID factors. 
This study used an accessible sampling method in which a particular group of participants is chosen for study because the researcher has easy access to them. All the informants were either in first and second-year degree programs in India. Participants were recruited from three public colleges, which include Government Degree College of Gulbarga, Ambedkar College, and the Central University of Karnataka and four private colleges which comprise of Reshmi College, Inamdar College, APPA College, and Gurukul College. To identify the three different groups of undergraduate whose past years of studying in a particular medium of instruction vary from one to another, informants were asked to specify in the questionnaire their previous language medium of education. These past years of learning are ranging from at least class 1 to class 10, class 11 to class 12, and first-year undergraduate. Three hundred fifty-eight students from different degree programs (Bachelor of Computer Application, Bachelor of Science, Bachelor of Commerce, Bachelor of Technology, and Bachelor of Arts) participated in this study. As it is displayed in Table 1, 97 respondents claimed that they began studying in English medium at either primary or high school which ranges from class 1 and class 10 (early immersion group of students). One hundred twenty-six informants started in class 11, which is the pre-university level (middle immersion group of students), while 135 respondents began the English medium of instruction at the undergraduate level (late immersion group of students).

Table 1: Profile of the samples in current study

\begin{tabular}{ccc}
\hline Immersion group & B.C.A/B.Sc/B.Com/B.Tech & B.A/B.Com \\
\hline $\begin{array}{c}\text { Early immersion group } \\
\text { of students }\end{array}$ & 97 & \\
$\begin{array}{c}\text { Middle immersion } \\
\text { group of students } \\
\text { Late immersion group } \\
\text { of students }\end{array}$ & 126 & \\
\hline Total & & 135 \\
\hline
\end{tabular}

A questionnaire was used to collect data for this study. It was designed from the review of related literature and the conceptual framework to measure the independent and dependent variables. Each item on it was scored on a fivepoint Likert scale with the following numerical values to indicate the degree to which respondents agreed or disagreed with the statement as follows: $5=$ Strongly Agree (SA); $4=$ Agree (A) $3=$ Neutral $(\mathrm{N}) ; 2=$ Disagree (D); $1=$ Strongly Disagree (SDA)

A pilot study was conducted at three Degree Colleges in Gulbarga City. It was used to assess the reliability of the instrument to gauge how long it will take the respondents to answer questions. It also helped the researcher to confirm whether items were stated clearly and had some meaning to all respondents.

The final data collection was done by first establishing rapport with respondents, explaining the purpose of the study, and the meaning of items that were not clear. Later, the exercise was carried out quietly in an organized manner, and the administration of the participating schools cooperated well 
with the researcher. The collected data were coded and analyzed using the Statistical Package for Social Sciences (SPSS) software.

\section{Results}

Table 2 depicts the order of ranking of all the motivational variables among early, middle, and late immersion students. In sum, the level of self-confidence, willingness to communicate, cultural interest, and parental factors declined from early to late immersion students. While language anxiety decreased from late immersion to early immersion students, late immersion students were very anxious to use the language. However, there is no significant difference among the three groups of immersions concerning the effect of intrinsic motivation, teachers' and peers' motivational influence. Lastly, the early immersions have the lowest level of motivational intensity even though they have the highest level of English proficiency.

Table: 2: Ranking of individual difference among early, middle and late immersion students

\begin{tabular}{|c|c|c|c|c|}
\hline ID Factors & Rank1 & Rank 2 & Rank 3 & Significance of Correlation \\
\hline Motivational intensity & Middle & Late & Early & $\begin{array}{l}\text { No significant difference between } \\
\text { late and middle }\end{array}$ \\
\hline Language anxiety & Late & Middle & Early & Significant difference \\
\hline Intrinsic motivation & Early & Middle & Late & $\begin{array}{l}\text { No Significant difference among the } \\
\text { three }\end{array}$ \\
\hline Self confidence & Early & Middle & Late & $\begin{array}{l}\text { No Significant difference between } \\
\text { middle and early }\end{array}$ \\
\hline WTC & Early & Middle & Late & $\begin{array}{l}\text { No Significant difference between } \\
\text { late and middle }\end{array}$ \\
\hline Cultural interest & Early & Middle & Late & $\begin{array}{l}\text { Significant difference among the } \\
\text { three }\end{array}$ \\
\hline $\begin{array}{l}\text { Instrumental } \\
\text { orientation }\end{array}$ & Late & Middle & Early & $\begin{array}{l}\text { No Significant difference among the } \\
\text { three }\end{array}$ \\
\hline Attitude & Early & Middle & Late & $\begin{array}{l}\text { No Significant difference between } \\
\text { late and middle }\end{array}$ \\
\hline Teachers factor & Late & Early & Late & $\begin{array}{l}\text { No Significant difference among the } \\
\text { three }\end{array}$ \\
\hline Parents & Early & Middle & Late & $\begin{array}{l}\text { Significant difference between late } \\
\text { and early }\end{array}$ \\
\hline Peers & Middle & Early & Late & $\begin{array}{l}\text { No Significant difference among the } \\
\text { three }\end{array}$ \\
\hline
\end{tabular}

Table 3 represents the correlation between motivational intensity and individual difference variable factors among early, middle, and late immersion students. In sum, there was a moderate correlation between motivational intensity and peers, parental factors, teachers' factors, self-confidence, intrinsic, and attitude towards English language education. In other words, all these motivational variable factors affected students' motivational intensity. 
Table 3: Correlation between motivational intensity and individual difference factors

\begin{tabular}{|c|c|c|c|c|c|c|}
\hline S/no & $\begin{array}{l}\text { Statement of } \\
\text { Correlation }\end{array}$ & $\begin{array}{c}\mathbf{R} \\
\text { value }\end{array}$ & $\begin{array}{l}\text { Strength } \\
\text { of } \\
\text { correlation }\end{array}$ & $\begin{array}{c}P \\
\text { value }\end{array}$ & $\begin{array}{l}\text { Nature of } \\
\text { correlation }\end{array}$ & $\begin{array}{c}\text { Significance } \\
\text { of } \\
\text { correlation }\end{array}$ \\
\hline 1 & $\begin{array}{l}\text { Motivational intensity and } \\
\text { attitude of immersion } \\
\text { Early immersion students }\end{array}$ & .214 & Very weak & .035 & Positive & Significant \\
\hline 2 & $\begin{array}{l}\text { Motivational intensity and } \\
\text { attitude of immersion } \\
\text { middle immersion students }\end{array}$ & .402 & Moderate & .000 & Positive & Significant \\
\hline 3 & $\begin{array}{l}\text { Motivational intensity and } \\
\text { attitude of late immersion } \\
\text { students }\end{array}$ & .274 & Weak & .001 & Positive & Significant \\
\hline 4 & $\begin{array}{l}\text { Motivational intensity and } \\
\text { instrumental motivation of } \\
\text { late immersion students }\end{array}$ & .332 & Moderate & .000 & Positive & Significant \\
\hline 5 & $\begin{array}{l}\text { Motivational intensity and } \\
\text { instrumental motivation of } \\
\text { middle immersion students }\end{array}$ & .467 & Moderate & .000 & Positive & Significant \\
\hline 6 & $\begin{array}{l}\text { Motivational intensity and } \\
\text { instrumental Motivation of } \\
\text { immersion early } \\
\text { immersion students }\end{array}$ & .273 & Weak & .007 & Positive & Significant \\
\hline 7 & $\begin{array}{l}\text { Motivational intensity and } \\
\text { cultural interest in } \\
\text { Kannada early immersion } \\
\text { students }\end{array}$ & .148 & Very weak & .147 & Positive & $\begin{array}{l}\text { Not } \\
\text { significant }\end{array}$ \\
\hline 8 & $\begin{array}{l}\text { Motivational intensity and } \\
\text { cultural interest in } \\
\text { Kannada middle } \\
\text { immersion students }\end{array}$ & 0.073 & None & .419 & Positive & $\begin{array}{l}\text { Not } \\
\text { significant }\end{array}$ \\
\hline 9 & $\begin{array}{l}\text { Motivational intensity and } \\
\text { cultural interest in } \\
\text { Kannada late immersion } \\
\text { students }\end{array}$ & -.235 & Weak & .006 & Negative & Significant \\
\hline 10 & $\begin{array}{l}\text { Motivational intensity and } \\
\text { intrinsic motivation late } \\
\text { immersion students }\end{array}$ & .307 & Weak & .000 & Positive & Significant \\
\hline 11 & $\begin{array}{l}\text { Motivational intensity and } \\
\text { intrinsic motivation early } \\
\text { immersion students }\end{array}$ & .3 & Weak & .003 & Positive & Significant \\
\hline 12 & $\begin{array}{l}\text { Motivational intensity and } \\
\text { intrinsic motivation middle } \\
\text { immersion students }\end{array}$ & .339 & Moderate & .000 & Positive & Significant \\
\hline 13 & $\begin{array}{l}\text { Motivational intensity and } \\
\text { self confidence among } \\
\text { middle immersion students }\end{array}$ & .097 & $\begin{array}{l}\text { No } \\
\text { correlation }\end{array}$ & .284 & Positive & $\begin{array}{l}\text { Not } \\
\text { significant }\end{array}$ \\
\hline 14 & $\begin{array}{l}\text { Motivational intensity and } \\
\text { self-confidence early } \\
\text { immersion students }\end{array}$ & -.014 & $\begin{array}{l}\text { No } \\
\text { correlation }\end{array}$ & .895 & Negative & $\begin{array}{l}\text { Not } \\
\text { significant }\end{array}$ \\
\hline 15 & $\begin{array}{l}\text { Motivational intensity and } \\
\text { self-confidence late }\end{array}$ & .354 & Moderate & .000 & Positive & Significant \\
\hline
\end{tabular}




\begin{tabular}{|c|c|c|c|c|c|c|}
\hline S/no & $\begin{array}{l}\text { Statement of } \\
\text { Correlation }\end{array}$ & $\begin{array}{c}\mathrm{R} \\
\text { value }\end{array}$ & $\begin{array}{l}\text { Strength } \\
\text { of } \\
\text { correlation }\end{array}$ & $\begin{array}{c}P \\
\text { value }\end{array}$ & $\begin{array}{l}\text { Nature of } \\
\text { correlation }\end{array}$ & $\begin{array}{l}\text { Significance } \\
\text { of } \\
\text { correlation }\end{array}$ \\
\hline 16 & $\begin{array}{l}\text { immersion students } \\
\text { Motivational intensity and } \\
\text { WTC late immersion } \\
\text { students }\end{array}$ & -1.54 & Strong & .075 & Negative & $\begin{array}{l}\text { Not } \\
\text { significant }\end{array}$ \\
\hline 17 & $\begin{array}{l}\text { Motivational intensity and } \\
\text { WTC middle immersion } \\
\text { students }\end{array}$ & -1.21 & Strong & .179 & Negative & $\begin{array}{l}\text { Not } \\
\text { significant }\end{array}$ \\
\hline 18 & $\begin{array}{l}\text { Motivational intensity and } \\
\text { WTC of early immersion } \\
\text { students }\end{array}$ & .208 & Weak & .041 & Positive & Significant \\
\hline 19 & $\begin{array}{l}\text { Motivational intensity and } \\
\text { anxiety of early immersion } \\
\text { students }\end{array}$ & .104 & Weak & .308 & Positive & $\begin{array}{l}\text { Not } \\
\text { significant }\end{array}$ \\
\hline 20 & $\begin{array}{l}\text { Motivational intensity and } \\
\text { anxiety middle immersion } \\
\text { students }\end{array}$ & .118 & Very weak & .191 & Positive & $\begin{array}{l}\text { Not } \\
\text { significant }\end{array}$ \\
\hline 21 & $\begin{array}{l}\text { Motivational intensity and } \\
\text { anxiety among late } \\
\text { immersion students }\end{array}$ & .109 & Very weak & 208 & Positive & $\begin{array}{l}\text { Not } \\
\text { significant }\end{array}$ \\
\hline 22 & $\begin{array}{l}\text { Motivational intensity and } \\
\text { teachers factors among } \\
\text { early immersion students }\end{array}$ & .120 & Weak & .242 & Positive & $\begin{array}{l}\text { Not } \\
\text { significant }\end{array}$ \\
\hline 23 & $\begin{array}{l}\text { Motivational intensity and } \\
\text { teachers factors among } \\
\text { middle immersion students }\end{array}$ & .332 & Moderate & .000 & Positive & Significant \\
\hline 24 & $\begin{array}{l}\text { Motivational intensity and } \\
\text { teachers factors among late } \\
\text { immersion students }\end{array}$ & .341 & Moderate & .000 & Positive & Significant \\
\hline 25 & $\begin{array}{l}\text { Motivational intensity and } \\
\text { parents factors among } \\
\text { immersion class } 11 \text { starter }\end{array}$ & .316 & Moderate & .000 & Positive & Significant \\
\hline 26 & $\begin{array}{l}\text { Motivational intensity and } \\
\text { parents factors among } \\
\text { early immersion students }\end{array}$ & .383 & Moderate & .054 & Positive & $\begin{array}{l}\text { Not } \\
\text { significant }\end{array}$ \\
\hline 27 & $\begin{array}{l}\text { Motivational intensity and } \\
\text { parents factors among late } \\
\text { immersion students }\end{array}$ & .267 & Moderate & .002 & Positive & Significant \\
\hline 28 & $\begin{array}{l}\text { Motivational intensity and } \\
\text { peers factors among } \\
\text { middle immersion students }\end{array}$ & .415 & Moderate & .000 & Positive & Significant \\
\hline 29 & $\begin{array}{l}\text { Motivational intensity and } \\
\text { peers factors early among } \\
\text { immersion students }\end{array}$ & ,361 & Moderate & .000 & Positive & Significant \\
\hline 30 & $\begin{array}{l}\text { Motivational intensity and } \\
\text { peers factors among late } \\
\text { immersion students }\end{array}$ & .194 & Weak & .096 & Positive & $\begin{array}{c}\text { Not } \\
\text { Significant }\end{array}$ \\
\hline
\end{tabular}

Table 4 shows a moderate correlation between affective variable factors (selfconfidence and anxiety) and English proficiency. But the rest ID factors are weakly correlated. 
Table 4: Correlation between English proficiency and individual difference factors

\begin{tabular}{|c|c|c|c|c|c|c|}
\hline n/o & Statement of Correlation & $\begin{array}{c}\mathbf{R} \\
\text { value }\end{array}$ & $\begin{array}{l}\text { Strength of } \\
\text { correlation }\end{array}$ & $\begin{array}{c}\mathbf{P} \\
\text { value }\end{array}$ & $\begin{array}{l}\text { Nature of } \\
\text { correlation }\end{array}$ & $\begin{array}{l}\text { Significance } \\
\quad \text { of } \\
\text { correlation }\end{array}$ \\
\hline 1 & $\begin{array}{l}\text { English proficiency and self } \\
\text { confidence among late immersion } \\
\text { students }\end{array}$ & .422 & Moderate & .000 & Positive & Significant \\
\hline 2 & $\begin{array}{l}\text { English proficiency and self } \\
\text { confidence among early } \\
\text { immersion students }\end{array}$ & .417 & Moderate & .000 & Positive & Significant \\
\hline 3 & $\begin{array}{l}\text { English proficiency and self- } \\
\text { confidence middle immersion } \\
\text { students }\end{array}$ & .249 & Weak & .005 & Positive & Significant \\
\hline 4 & $\begin{array}{l}\text { English proficiency and anxiety } \\
\text { among late immersion students }\end{array}$ & .22 & Weak & .010 & Positive & Significant \\
\hline 5 & $\begin{array}{l}\text { English proficiency and anxiety } \\
\text { among early immersion students }\end{array}$ & .201 & Weak & .048 & Positive & Significant \\
\hline 6 & $\begin{array}{l}\text { English proficiency and anxiety } \\
\text { among middle immersion students }\end{array}$ & .152 & Very weak & .091 & Positive & $\begin{array}{l}\text { Not } \\
\text { significant }\end{array}$ \\
\hline 7 & $\begin{array}{l}\text { English proficiency and WTC } \\
\text { among late immersion students }\end{array}$ & -.238 & Weak & .006 & Negative & Significant \\
\hline 8 & $\begin{array}{l}\text { English proficiency and WTC } \\
\text { among middle immersion students }\end{array}$ & -.166 & Very weak & .064 & Negative & Significant \\
\hline 9 & $\begin{array}{l}\text { English proficiency and WTC } \\
\text { among early immersion students }\end{array}$ & -.254 & Weak & .012 & Negative & Significant \\
\hline 10 & $\begin{array}{l}\text { English proficiency and cultural } \\
\text { interest among late immersion } \\
\text { students }\end{array}$ & -.175 & Very weak & .043 & Negative & Significant \\
\hline 11 & $\begin{array}{l}\text { English proficiency and cultural } \\
\text { interest among middle immersion } \\
\text { students }\end{array}$ & -.122 & Very weak & .174 & Negative & $\begin{array}{l}\text { Not } \\
\text { significant }\end{array}$ \\
\hline 12 & $\begin{array}{l}\text { English proficiency and cultural } \\
\text { interest among early immersion } \\
\text { students }\end{array}$ & -.202 & Weak & .047 & Negative & Significant \\
\hline 13 & $\begin{array}{l}\text { English proficiency and enjoy } \\
\text { among late immersion students }\end{array}$ & .072 & Very weak & .405 & Positive & $\begin{array}{l}\text { Not } \\
\text { significant }\end{array}$ \\
\hline 14 & $\begin{array}{l}\text { English proficiency and enjoy } \\
\text { among middle immersion students }\end{array}$ & .328 & Moderate & .000 & Positive & Significant \\
\hline 15 & $\begin{array}{l}\text { English proficiency and enjoy } \\
\text { among early immersion students } \\
\text { English proficiency and }\end{array}$ & .154 & Very weak & .131 & Positive & $\begin{array}{c}\text { Not } \\
\text { significant }\end{array}$ \\
\hline 16 & $\begin{array}{l}\text { instrumental early immersion } \\
\text { students }\end{array}$ & .254 & Weak & .012 & Positive & Significant \\
\hline 17 & $\begin{array}{l}\text { English proficiency and } \\
\text { instrumental middle immersion } \\
\text { students }\end{array}$ & .152 & Very weak & .091 & Positive & $\begin{array}{l}\text { Not } \\
\text { significant }\end{array}$ \\
\hline 18 & $\begin{array}{l}\text { English proficiency and } \\
\text { instrumental among late } \\
\text { immersion students }\end{array}$ & .073 & None & .401 & Positive & $\begin{array}{l}\text { Not } \\
\text { significant }\end{array}$ \\
\hline 19 & $\begin{array}{l}\text { English proficiency and attitude } \\
\text { late immersion students }\end{array}$ & -.053 & None & .541 & Negative & $\begin{array}{l}\text { Not } \\
\text { significant }\end{array}$ \\
\hline 20 & $\begin{array}{l}\text { English proficiency and attitude } \\
\text { among middle immersion students }\end{array}$ & .046 & None & .607 & Positive & $\begin{array}{c}\text { Not } \\
\text { significant }\end{array}$ \\
\hline
\end{tabular}




\begin{tabular}{|c|c|c|c|c|c|c|}
\hline n/o & Statement of Correlation & $\begin{array}{c}\mathbf{R} \\
\text { value }\end{array}$ & $\begin{array}{l}\text { Strength of } \\
\text { correlation }\end{array}$ & $\begin{array}{c}P \\
\text { value }\end{array}$ & $\begin{array}{l}\text { Nature of } \\
\text { correlation }\end{array}$ & $\begin{array}{l}\text { Significance } \\
\quad \text { of } \\
\text { correlation }\end{array}$ \\
\hline 21 & $\begin{array}{l}\text { English proficiency and attitude } \\
\text { among early immersion students }\end{array}$ & .104 & Very weak & .309 & Positive & $\begin{array}{c}\text { Not } \\
\text { significant }\end{array}$ \\
\hline 22 & $\begin{array}{l}\text { English proficiency and parents } \\
\text { among early immersion students }\end{array}$ & .183 & Very weak & .370 & Positive & $\begin{array}{l}\text { Not } \\
\text { significant }\end{array}$ \\
\hline 23 & $\begin{array}{l}\text { English proficiency and parents } \\
\text { among middle immersion students }\end{array}$ & .203 & Weak & .023 & Positive & Significant \\
\hline 24 & $\begin{array}{l}\text { English proficiency and parents } \\
\text { among late immersion students }\end{array}$ & -.027 & None & .758 & Negative & $\begin{array}{l}\text { Not } \\
\text { significant }\end{array}$ \\
\hline 25 & $\begin{array}{l}\text { English proficiency and teachers } \\
\text { among late immersion students }\end{array}$ & .18 & Weak & .036 & Positive & Significant \\
\hline 26 & $\begin{array}{l}\text { English proficiency and teachers } \\
\text { middle immersion students }\end{array}$ & .113 & Weak & .210 & Positive & $\begin{array}{l}\text { Not } \\
\text { significant }\end{array}$ \\
\hline 27 & $\begin{array}{l}\text { English proficiency and teachers } \\
\text { among early immersion students }\end{array}$ & .202 & Weak & .048 & Positive & Significant \\
\hline 28 & $\begin{array}{l}\text { English proficiency and peers } \\
\text { among early immersion students }\end{array}$ & .149 & Very weak & .148 & Positive & $\begin{array}{l}\text { Not } \\
\text { significant }\end{array}$ \\
\hline 29 & $\begin{array}{l}\text { English proficiency and peers } \\
\text { among middle immersion students }\end{array}$ & .144 & Very weak & .109 & Positive & $\begin{array}{c}\text { Not } \\
\text { significant }\end{array}$ \\
\hline 30 & $\begin{array}{l}\text { English proficiency and peers late } \\
\text { immersion students }\end{array}$ & .166 & Very weak & .054 & Positive & $\begin{array}{l}\text { Not } \\
\text { significant }\end{array}$ \\
\hline 31 & $\begin{array}{l}\text { Motivational intensity and English } \\
\text { proficiency of among late } \\
\text { immersion students }\end{array}$ & .16 & Very weak & .064 & Positive & $\begin{array}{l}\text { Not } \\
\text { significant }\end{array}$ \\
\hline 32 & $\begin{array}{l}\text { Motivational intensity and English } \\
\text { proficiency among middle } \\
\text { immersion students }\end{array}$ & .102 & Very weak & .256 & Positive & $\begin{array}{l}\text { Not } \\
\text { significant }\end{array}$ \\
\hline 33 & $\begin{array}{l}\text { Motivational intensity and English } \\
\text { proficiency among early } \\
\text { immersion students }\end{array}$ & -.014 & Very weak & .894 & Negative & $\begin{array}{l}\text { Not } \\
\text { significant }\end{array}$ \\
\hline
\end{tabular}

\section{Discussion}

This study set out to investigate the role of ID factors in L2 learning among early, middle, and late immersion students. Thus, the study aims to answer several research questions such as the impact of every ID factor, degree of correlation between motivational intensity and other ID factors, and connection between ID factors and English proficiency among the three groups of immersion. The most striking result from the data is that the middle immersion students had the highest level of motivational intensity (3.784), followed by late immersion students (3.6716) while the group of students with the lowest level of motivational intensity (3.3814) was reported among early immersion students. In other words, the majority of early immersion students did not spend much time studying English or preparing very hard for its examination, unlike the other two immersion groups. A plausible explanation for this is that most early immersion learners considered themselves to be highly proficient in English. Therefore, the learners did not have any compelling reasons to expend much effort in learning the language. Also, the priority of average fresh undergraduate learners from English medium backgrounds tends 
to change from English language learning to learning of other courses which are also taught in English.

Regarding the role of anxiety among the three groups, late immersion students were found to have the highest level of anxiety, followed by middle immersion students, while the least form of anxiety was recorded among early immersion students. In other words, the higher the number of years of learning English across all curricula, the lower the level of anxiety towards speaking and writing of English exam. A possible explanation for this result may be due to communication apprehensiveness, which was reported to be common among the late immersion students (Mustapha, 2010).

With respect to self-confidence, the ID factor decreased progressively from early to Middle and then to Late immersion students. This finding suggests that the degree of self-confidence in passing English examinations and speaking English in public corresponds to the years of studying in English across the curriculum. This result may be explained by the fact that learning English across the curriculum tends to increase learners' self-confidence in the target language because early immersion students do not only learn in English class but also while learning other core subjects.

Similarly, WTC decreased from early to middle and then late immersion students. This high propensity in the usage of English might be due to convenient usage of the language, which is as a result of learning English across all curriculums. Furthermore, the reason can be attributed to the 6th layer of WTC pyramid propounded by (Macintyre, Dörnyei, Clément, \& Noels, 1998).

As far as the concept of instrumental motivation to learn L2 is concerned, the result showed that all the students in the three immersion groups had high instrumental motivation. That is, they acknowledged the importance of learning English to get a job easily, getting more knowledge, and earning respect. The finding of this study mirrors those of past studies that supported the strong existence of instrumental orientation in countries where English is regarded as a second or international language (Hong \& Ganapathy, 2017).

Also the role of teachers as a motivational agent was reaffirmed. The majority of the students in all the three groups $(84.4 \%, 81.4 \%$, and $76.8 \%)$ claimed that their English teachers motivate and encourage them to learn English. But parental motivations were higher among early and middle immersion group. These can be attributed to the low socioeconomic status of late immersion groups whose parents are not highly educated. Therefore, it is more likely for the parents not to show interest in their children's performance in English.

All the three immersion groups derived internal pleasure in learning and speaking the language. Interestingly, the majority of late immersion students (4.1556) claimed that they enjoyed using English outside the classroom whenever they have the chance to do so. This high level of internal disposition 
towards the language is due to the feelings of pleasure derived from fulfilling innate needs for competence and self-determination (Rahmanpanah, 2017).

The relationship between motivational intensity and teachers' motivational factors demonstrated a moderate positive correlation among middle (.332) and late immersion students (.341), but a weak positive correlation was observed among early immersion students (.12). This mild weak positive correlation indicates that the more teachers motivate their students to learn, the more students' motivational intensity is enhanced. This enhancement, which is more evident among middle and late immersion, maybe due to extra attention given by teachers, considering the students fewer years of the learning experience.

Concerning the connection between the parental motivational influence and students' motivational intensity, the results showed that parental motivational affect learners' motivational intensity, and this is prominent among early and middle immersion students. The correlation is even further enhanced when both parents are proficient in English. The resultant effect of this is that the students have a higher frequency rate of using the target language both in the classroom and at home.

With respect to the degree of peer influence on students' motivational intensity, a moderate positive correlation was recorded among early (.361) and middle immersion learners (.415). The main possible explanation for this may be anchored on the fact that early immersion students spend more years in an immersion program than late immersion students. That is, early and middle immersion learners have more years to improve one another English language achievement and proficiency because English is thriving in their learning environment, unlike late immersion students where partial immersion dominated the classroom medium of instruction. Consequently, early immersion students tend to develop the attitude of helping one another in spoken English or any task given by their teachers.

On the other hand, a high level of self-confidence among the early and middle immersion students is revealed in this study. Surprisingly, there is no correlation between the two variables early (.014) and middle immersion students (.097). However, a moderate positive relationship was observed among late immersion students (.354). It is convenient to argue that the lack of relationship between the latter is a result of the high level of self-confidence recorded in the earlier findings of this study. Certainly, an over-confidence student does not have any reason to work hard or invest so much time in an activity that is not considered challenging.

In the case of cultural interest, non-correlation between motivational intensity and cultural interest is feasible among late (.-235) and middle immersion students (.0073). This non-correlation is an indication that cultural materials do not motivate students to learn English. 
Conversely, early immersion students showed a weak negative correlation between the two variables. That is, the higher their motivational intensity to learn English, the lower their interest in Kannada cultural materials. This phenomenon may be because early immersion students have developed more interest in English cultural materials than the other immersion group of students over the years. This interest has compelled them to think, read, write, and speak more in English than in their mother tongues, which consequently raise their interest in English cultural materials rather than that of their mother tongue.

Despite high motivational intensity reported among middle and late immersion learners, a weak correlation was observed between English proficiency and motivational intensity variables. Also, there is no significant difference among the three immersion groups. It means that the level of effort put into learning the language does not highly correspond to the level of English proficiency. It is difficult to explain this result. Nevertheless, it might be related to the fact that other predictors such as anxiety and other impeding factors are capable of preventing learners from enhancing their proficiency.

Regarding the influence of self-confidence on English proficiency, a moderate correlation was recorded among early (.417) and late immersion students (.422), while the weak correlation was reported among middle immersion students (.249). It shows that immersion enhances students' self-confidence in the language, which in turn improves their English proficiency. This self-confidence must have stemmed from the fact that they believe in their ability to achieve any task in the English language. The ability is sharpening for early immersion students because immersion in English probably gives them more avenues to use the language, thereby perfect their proficiency over the years. On the other hand, the weak positive correlation among the three groups of immersion students implies that anxiety has a weak debilitative effect on the immersion students.

Meanwhile, the relationship between cultural interest and English proficiency, a significant weak negative correlation was found across the three groups of immersion students. Taking into account that cultural interest in Kannada is inversely related to English proficiency in the Indian context. In simple terms, if learners' cultural interest in Kannada increases, then English proficiency is decreased and vice versa. This finding is in line with past works that have reported the positive effect of cultural interest on English proficiency. It suggests that immersion programs trigger learners' interest in cultural material, thereby enhancing their English ability.

Lastly, among teachers, parents, and peers motivational influence, a correlation between the two variables among middle, late, and early immersion students were revealed while there was no significant relationship between English proficiency and peers motivational influence. The findings show that parents and teachers play a huge role in influencing students in enhancing English proficiency. The correlation was reported even among late immersion students. This occurrence is surprising because these groups of students are products of 
partial immersion lessons. Partial immersion teachers are a group of educators who are compelled to code-switching during teaching because of low proficiency students in the class. Hence, reporting a positive correlation among late immersion students gives credence to the premise that human factors, in this case, teachers, play a significant contribution to the language proficiency of students.

\section{Conclusion}

The main goal of the current study was to determine the role of ID factors among early, middle, and late immersion undergraduate ESL learners. Determination of the level of motivational intensity and other individual difference factors were used to answer the first research question. Early immersion students were found to have the lowest form of motivational intensity among the three groups of immersion. This finding is a consequence of the fact that English learning is not early immersion students' priority at an undergraduate level. In addition, early immersion students were the group of students that have the highest level of self-confidence, interest in English cultural materials as well as English language as a whole, strong positive attitude towards English language education. They are the group of students mostly motivated and encouraged by their parents to learn English while late immersion students possess the highest level of language anxiety and the most highly instrumental motivated group. These findings shed light on the significant factors responsible for the highest English proficiency reported among early immersion students. In addition, this study provided the answer to the second research question by reporting a moderate and higher significant relationship between motivational intensity and other motivational variable factors such as attitude towards English education and its status, self-confidence, teachers', parents' and peers' motivational influence. This moderate correlation existed both among middle and late immersion students. Thus, it could be concluded that these factors are responsible for higher motivational intensity reported among middle and late immersion students. However, unlike motivational intensity, only self-confidence and intrinsic motivation have a direct moderate correlation with English proficiency. It is the answer to the third research question. Surprisingly, there is no significant relationship between motivational intensity and English proficiency. Contrary to previous findings, effort rarely translates to performance in L2 because the motivational intensity of the learners stems from writing of English exam.

The overall findings of this study suggest that difference in the past language learning experience of Undergraduate ESL learners influences their ID factors. This work contributes to existing knowledge of ID factors by establishing its significance in English language immersion.

These findings imply that scholars and researchers need to take into cognizance of the effect of the past medium of instruction of their informants, especially whenever there is a need to measure any motivational variable among EFL undergraduates. For teachers, it is important to be mindful of the different backgrounds of their students by firstly identify the nature of immersion of their students, considering that their level of proficiency might vary from one 
another. It will help them in deploying a proper teaching methodology in such a mixed classroom

\section{References}

Ababneh, S. (2013). The effects of motivation on female EFL students' language proficiency. Journal of Education and Practice, 4(7), 118-124.

Aljasir, N. (2016). Individual differences among Saudi learners of English as a foreign language: an exploratory correlational study of learning styles, affective factors and English proficiency and performance (Doctoral dissertation, University of Birmingham).

Alrajhi, M. N., \& Aldhafri, S. S. (2015). Peer Tutoring Effects on Omani Students' English $\begin{array}{lllll}\text { Self-Concept. International Education Studies, } & 8(6), & 184 .\end{array}$ http://doi:10.5539/ies.v8n6p184

Anand, C. L. (1971). The Study of the Effect of Socio-economic Environment and Medium of Instruction on the Mental Abilities and Academic Achievement of Children in Mysore State (Unpublished Doctoral dissertation, University of Mysore, Mysore).

Andrade, M., \& Williams, K. (2009). Foreign Language Learning Anxiety in Japanese EFL University Classes: Physical, Emotional, Expressive, and Verbal Reactions. Sophia Junior College Faculty Journal, 29, 1-24.

Basta, J. (2011). The role of the communicative approach and cooperative learning in higher education. Facta Universitatis- Series: Linguistics and Literature, 9(2), 125144.

Dornyei, Z. (2001). Teaching and Researching Motivation. Applied Linguistics in Action. http://doi:10.4324/9781315833750

Entwislea, D. R., \& Astone, N. M. (1994). Some practical guidelines for measuring youth's race/ethnicity and socioeconomic status. Child Development, 65(6), 15211540. http://dx.doi.org/10.2307/1131278

Fachtmann, R., Fountaine, S., Grove, R., Hoshino, B., Jensen, D., Kiak, L. S., ... \& Matsuda, S. (2001). Personality, motivation, anxiety, strategies, and language proficiency of Japanese students. Motivation and second language acquisition, 23, 361.

Firdosfida, M., \& Ravindra, K. (2016). Relationship between students' motivation, attitude and motivational intensity toward English learning, 5(2), 27-33.

Gardner, R. C. (1985). Social psychology and second language learning: The role of attitudes and motivation. Arnold. https:// doi.org/10.1017/S0272263100007634

Gayton, A. (2010). Socioeconomic status and language-learning motivation: To what extent does the former influence the latter? Scottish Languages Review, 22(1), 1728.

Gregersen, T. (2000). The individual differences that distinguish high and low output students. Revistasignos, 33(47), 87-99. http://dx.doi.org/10.4067/S071809342000000100008

Guilloteaux, M. J., \& Dornyei, Z. (2008). Motivating Language Learners: A ClassroomOriented Investigation of the Effects of Motivational Strategies on Student Motivation. TESOL Quarterly: A Journal for Teachers of English to Speakers of Other Languages and of Standard English as a Second Dialect, 42(1), 55-77. http://doi.org/10.1002/j.1545-7249.2008.tb00207.x

Henkel, B. (2009). Motivation to learn a second language for minority students. Practice and Theory in Systems of Education, 4(2), 63-78. 
Hiromori, T., Matsumoto, H., \& Nakayama, A. (2012). Profiling individual differences of successful and unsuccessful L2 readers. The Journal of AsiaTEFL, 9(2), 49-70. http://doi.org/10.1016/j.lindif.2012.03.009

Hong, Y. C., \& Ganapathy, M. (2017). To Investigate ESL Students' Instrumental and Integrative Motivation towards English Language Learning in a Chinese School in Penang: Case Study. English Language Teaching, 10(9), 17-35.

Johari, A. (2015, July 30). Taunting, teasing, segregation: What regional-language students face in English-medium colleges? Scroll.in.

Khodadady, E., \& Khajavy, G. H. (2013). Exploring the Role of Anxiety and Motivation in Foreign Language Achievement: a Structural Equation Modeling Approach. Porta Linguarum: Revista Internacional de Didáctica de Las Lenguas Extranjeras, (20), 269-286.

Kidd, E., Donnelly, S., \& Christiansen, M. H. (2018). Individual differences in language acquisition and processing. Trends in cognitive sciences, 22(2), 154-169. https://doi:10.1016/j.tics.2017.11.006

Lahuerta, A. C. (2014). Factors affecting willingness to communicate in a Spanish university context. IJES, International Journal of English Studies, 14(2), 39-55. https://doi.org/10.6018/j.193611

Lasekan, O. A. (2019). Self-Assessment of English Proficiency among Early, Middle and Late Immersion Undergraduate Students. In Basavaraja Kodagunti (Ed.), Papers on Current Issues in Indian Linguistics (pp. 55-64). Maski, India: Bandara Parkashana India.

MacIntyre, P. D., Dörnyei, Z., Clément, R., \& Noels, K. A. (1998). Conceptualizing willingness to communicate in a L2: A situational model of L2 confidence and affiliation. The Modern Language Journal, 82(4), 545-562. http://doi:10.1111/j.15404781.1998.tb05543.x.

McCroskey, J. C., \& Richmond, V. P. (1990). Willingness to communicate: Differing cultural perspectives. Southern Communication Journal, 56(August 2014), 72-77 http://doi.org/10.1080/10417949009372817

Mustapha, W. Z. W. (2010). ESL students' communication apprehension and their choice of communicative activities. Asean Journal of Teaching and Learning in Higher Education (AJTLHE), 2(1), 22-29.

Oroujlou, N., \& Vahedi, M. (2011). Motivation, attitude, and language learning. In Procedia -Social and Behavioral Sciences (Vol. 29, pp. 994 1000). http://doi.org/10.1016/j.sbspro.2011.11.333

Oxford, R. L., \& Ehrman, M. (1992). Second language research on individual differences. Annual review of applied linguistics, 13, 188-205. https://doi.org/10.1017/S0267190500002464

Pintrich, P. R., \& Schunk, D. H. (2002). Motivation in education: Theory, research, and applications. Prentice Hall.

Rahmanpanah, H. (2017). Self-determination Theory as a Grand Theory of Motivation in EFL Classroom. Journal of Applied Linguistics and Language Research, 4(6), 153-164.

Robinson, P. (Ed.). (2002). Individual differences and instructed language learning (Vol. 2). John Benjamins Publishing. https://doi.org/10.1075/1llt.2.13rob

Satriani, I. (2014). Correlation between Students' Self Esteem and English Language Proficiency of Indonesian EFL Students. Journal of English Language Teaching in Indonesia, 2(2). https://doi.org/10.22460/eltin.v2i2.p\%25p 